\title{
Erratum: Emergent Supersymmetry from Strongly Interacting Majorana Zero Modes
} [Phys. Rev. Lett. 115, 166401 (2015)]

Armin Rahmani, Xiaoyu Zhu, Marcel Franz, and Ian Affleck (Received 12 February 2016; published 9 March 2016)

DOI: 10.1103/PhysRevLett.116.109901

The following statement was missing from the acknowledgements: This research was enabled, in part, by support provided by WestGrid and Compute Canada Calcul Canada. 